\title{
Linear ac response of diffusive SNS junctions
}

\author{
Pauli Virtanen, ${ }^{1,2}$ F. Sebastián Bergeret, ${ }^{3,4}$ Juan Carlos Cuevas, ${ }^{5}$ and Tero T. Heikkilä ${ }^{2}$ \\ ${ }^{1}$ Institute for Theoretical Physics and Astrophysics, University of Würzburg, D-97074 Würzburg, Germany \\ ${ }^{2}$ Low Temperature Laboratory, Aalto University, P.O. Box 15100, FI-00076 Aalto, Finland \\ ${ }^{3}$ Centro de Física de Materiales (CFM), Centro Mixto CSIC-UPV/EHU, Edificio Korta, Avenida de Tolosa 72, E-20018 San Sebastián, Spain \\ ${ }^{4}$ Donostia International Physics Center (DIPC), Manuel de Lardizbal 4, E-20018 San Sebastián, Spain \\ ${ }^{5}$ Departamento de Física Teórica de la Materia Condensada, Universidad Autónoma de Madrid, E-28049 Madrid, Spain
}

(Received 20 December 2010; revised manuscript received 17 February 2011; published 19 April 2011)

\begin{abstract}
We explore the behavior of the ac admittance of superconductor-normal-metal-superconductor (SNS) junctions as the phase difference of the order parameters between the superconductors is varied. We find three characteristic regimes, defined by comparison of the driving frequency $\omega$ to the inelastic scattering rate $\Gamma$ and the Thouless energy $E_{T}$ of the junction (typically $\hbar \Gamma \ll E_{T}$ ). Only in the first regime $\omega \ll \Gamma$ does the usual picture of the kinetic inductance hold. We show that the ac admittance can be used to directly access some of the characteristic quantities of the SNS junctions, in particular the phase-dependent energy minigap and the typically phase-dependent inelastic scattering rate. Our results partially explain the recent measurements of the linear response properties of SNS superconducting quantum interference devices (SQUIDs) and predict a number of new effects.
\end{abstract}

DOI: 10.1103/PhysRevB.83.144514

PACS number(s): 74.45.+c, 74.25.N-, 74.50.+r

\section{INTRODUCTION}

The frequency-dependent susceptibility typically reveals information about the internal dynamics of the studied systems. In the electronic case, this susceptibility is more often measured as admittance, whose frequency dependence in semiclassical models for bulk metals is due to scattering and appears for frequencies exceeding some tens of terahertz. ${ }^{1}$ In wires, it is dictated by stray capacitances and geometric inductance. Understanding the frequency-dependent response is moreover of importance for high-frequency devices.

In superconductors or superconducting tunnel junctions (STJs), the admittance at low frequencies is dominated by the superconducting kinetic inductance. ${ }^{2}$ For STJs, the Josephson inductance is related to the supercurrent $I_{S}(\varphi)$ through the junction, which depends on the superconducting phase difference $\varphi$ :

$$
L_{J}(\varphi)^{-1}=\frac{2 e}{\hbar} \partial_{\varphi} I_{S}(\varphi) .
$$

This relation allows characterization of the current-phase relation of Josephson junctions via measurements of their ac admittance. ${ }^{3}$ The remaining dissipative part of the admittance is due to quasiparticles, is proportional to $\exp [-\mid \Delta-$ $\left.\hbar \omega \mid /\left(k_{B} T\right)\right],{ }^{4}$ and is important only for the high frequencies of the order of the superconducting gap $\Delta$ or temperatures $T$ close to the critical temperature.

For Josephson junction types other than STJs, however, the admittance can deviate from the above simple picture. In this article we show how combining normal-metal wires $(\mathrm{N})$ and superconductors (S) into superconductor-normal-metalsuperconductor (SNS) weak links results in an admittance that entails characteristics of the inelastic scattering rates and the inverse diffusion times through the structure. At frequencies of the order of or larger than the inelastic scattering rate $\Gamma$, the simple kinetic-inductance picture has to be revised to include nonadiabatic effects associated with the dynamics of the electron energy distribution. The dissipative response, describing microwave absorption, is moreover finite for temperatures or frequencies exceeding the phase-dependent minigap in the spectrum of excitations inside the junction. The dissipative part of the admittance probes the density of states in the junction and is related to the physics of stimulation and suppression of the supercurrent. ${ }^{5,6}$

This paper is organized as follows. We first introduce in Sec. II the linear-response Keldysh approach we use to describe the admittance. After that, in the first part of Sec. III, we derive a number of analytical results applicable at frequencies low compared to $E_{T} / \hbar$, and we discuss the general low-frequency characteristics. The second part of Sec. III completes the picture by presenting results applicable at higher frequencies. Section IV concludes the paper.

\section{MODEL}

We study diffusive SNS junctions, whose length $L$ is longer than the superconducting coherence length $\xi_{0}=\sqrt{\hbar D / \Delta}$, where $D$ is the diffusion constant. The proximity effect induces a gap in the density of states inside the normal metal, $E_{g}(\varphi=0)=3.12 E_{T} \ll \Delta$, where $E_{T}=\hbar D / L^{2}$ is the inverse diffusion time. The minigap depends on the phase difference approximately as $\cos (\varphi / 2)$ and vanishes for $\varphi=\pi$. We model the coupling to the electric field with an ac bias voltage $V(t)=\delta V \sin (\omega t)$, which induces an oscillating phase difference $\varphi(t)=\varphi+\delta \phi \cos (\omega t)$ across the junction.

To find quantitative results, we describe the SNS junction dynamics with the Keldysh-Usadel equation, ${ }^{7}$ used also in Ref. 6. In this approach, physical quantities are obtained from the retarded, advanced, and Keldysh Green's functions $\hat{g}^{R / A / K}\left(E, E^{\prime}\right)$, which depend on two energy arguments. These functions are matrices in the Nambu (electronhole) space, and the Keldysh $(\mathrm{K})$ part can be parameterized in terms of an electron distribution function matrix $\hat{h}\left(E, E^{\prime}\right)=h_{L}\left(E, E^{\prime}\right)+h_{T}\left(E, E^{\prime}\right) \hat{\tau}_{3}: \hat{g}^{K}=\hat{g}^{R} \hat{h}-\hat{h} \hat{g}^{A}$. The matrix products also involve convolutions: $(\hat{B} \hat{C})\left(E, E^{\prime}\right)=$ $\int_{-\infty}^{\infty} \frac{d E_{1}}{2 \pi} \hat{B}\left(E, E_{1}\right) \hat{C}\left(E_{1}, E^{\prime}\right)$. In the presence of the harmonic 
drive, these functions can be written in a matrix representation ${ }^{8}$ $\hat{g}_{n, m}(E) \hat{=} \hat{g}(E+n \hbar \omega, E+m \hbar \omega)$ that reduces convolutions to matrix products.

For what follows, it is convenient to write the timedependent Usadel equation in the form

$$
\begin{gathered}
\hbar D \hat{\partial}_{x} \hat{j}^{R / A}=\left[-i \epsilon \hat{\tau}_{3}+i U+i \hat{\sigma}^{R / A}, \hat{g}^{R / A}\right], \\
\hat{g}^{R / A} \hat{g}^{R / A}=\hat{1}, \\
\hat{j}^{R / A} \equiv \hat{g}^{R / A} \hat{\partial}_{x} \hat{g}^{R / A}, \\
\hbar D \hat{\partial}_{x} \hat{j}^{K}=\left(\hat{\partial}_{x} \hat{j}^{R}\right) \hat{h}-\hat{h}\left(\hat{\partial}_{x} \hat{j}^{A}\right)+\hat{g}^{R} i \hat{Z}-i \hat{Z} \hat{g}^{A}, \\
\hat{j}^{K} \equiv \hat{j}^{R} \hat{h}-\hat{h} \hat{j}^{A}+\hat{\partial}_{x} \hat{h}-\hat{g}^{R}\left(\hat{\partial}_{x} \hat{h}\right) \hat{g}^{A}, \\
\hat{Z} \equiv\left[-\epsilon \hat{\tau}_{3}+U, \hat{h}\right]+\hat{\sigma}^{R} \hat{h}-\hat{h} \hat{\sigma}^{A}-\hat{\sigma}^{K},
\end{gathered}
$$

where $\epsilon\left(E, E^{\prime}\right) \equiv \delta\left(E-E^{\prime}\right) E, x$ is the position along the junction, $\hat{\partial}_{x} \hat{B}=\partial_{x} \hat{B}-i\left[A \hat{\tau}_{3}, \hat{B}\right]$ the gauge-covariant gradient containing the vector potential $A\left(E, E^{\prime}\right)=A(E) \delta\left(E-E^{\prime}\right)$, $D$ is the diffusion constant, and $U$ is the scalar potential. The self-energy $\hat{\sigma}^{R / A / K}$ describes relaxation processes inside the junction: we make a relaxation time approximation, taking $\hat{\sigma}^{R / A}=\mp i \hbar \Gamma \hat{\tau}_{3}, \hat{\sigma}^{K}=\hat{\sigma}^{R} \hat{h}_{\mathrm{eq}}-\hat{h}_{\mathrm{eq}} \hat{\sigma}^{A}$, where $\Gamma$ is an inelastic relaxation rate. We moreover assume clean boundary conditions: $\hat{g}^{R / A / K}$ are continuous across the N/S interfaces, and inside the superconductors they obtain their equilibrium BCS values.

Given solutions to Eqs. (2a)-(2f), the ac admittance can be obtained from the ac current harmonics via

$$
\begin{gathered}
Y(\omega)=\frac{2}{i \omega \delta \phi} I(\omega), \\
I(\omega)=\frac{\sigma_{N} S}{4} \int_{-\infty}^{\infty} d E \operatorname{Tr} \hat{\tau}_{3} \hat{j}_{01}^{K}(E),
\end{gathered}
$$

under a linear-response drive $\delta \phi \ll 2 \pi$. Here, $\sigma_{N}$ and $S$ are the normal-state conductivity and the cross section of the junction, respectively.

\section{ADMITTANCE}

In the above approach, the admittance splits naturally into three gauge-invariant parts, $Y=Y_{\mathrm{sc}}+Y_{\mathrm{dy}}+Y_{\mathrm{qp}}$, where (hereafter, $\hbar=e=k_{B}=1$ )

$$
\begin{gathered}
Y_{\mathrm{sc}}=\frac{\sigma_{N} S}{2 i \omega \delta \phi} \int_{-\infty}^{\infty} d E \operatorname{Tr}\left[\hat{\tau}_{3}\left(\hat{j}_{01}^{R} h_{11}-h_{00} \hat{j}_{01}^{A}\right)\right], \\
Y_{\mathrm{dy}}=\frac{\sigma_{N} S}{2 i \omega \delta \phi} \int_{-\infty}^{\infty} d E \operatorname{Tr}\left[\hat{\tau}_{3}\left(\hat{j}_{00}^{R} \hat{h}_{01}-\hat{h}_{01} \hat{j}_{11}^{A}\right)\right], \\
Y_{\mathrm{qp}}=\frac{\sigma_{N} S}{2 i \omega \delta \phi} \int_{-\infty}^{\infty} d E \operatorname{Tr}\left\{\left(1-\hat{\tau}_{3} \hat{g}_{11}^{A} \hat{\tau}_{3} \hat{g}_{00}^{R}\right)\right. \\
\left.\times\left[\hat{\tau}_{3} \partial_{x} \hat{h}_{01}-\frac{i A_{0}}{2}\left(h_{11}-h_{00}\right)\right]\right\} .
\end{gathered}
$$

Here $\hat{j}^{R / A}=\hat{g}^{R / A} \hat{\partial}_{x} \hat{g}^{R / A}$ describes spectral (super)currents, $h_{00}(E)=h_{11}(E-\omega)=\tanh (E / 2 T)$ is the electron energy distribution at equilibrium, and $\hat{h}_{01}$ is its time-dependent part. The contributions describe (a) the ac supercurrent, (b) the effect of the dynamic variation of the populations of the Andreev levels, and (c) the quasiparticle current driven directly by the field. Below we work mostly in a gauge in which the electric field is contained in the vector potential:

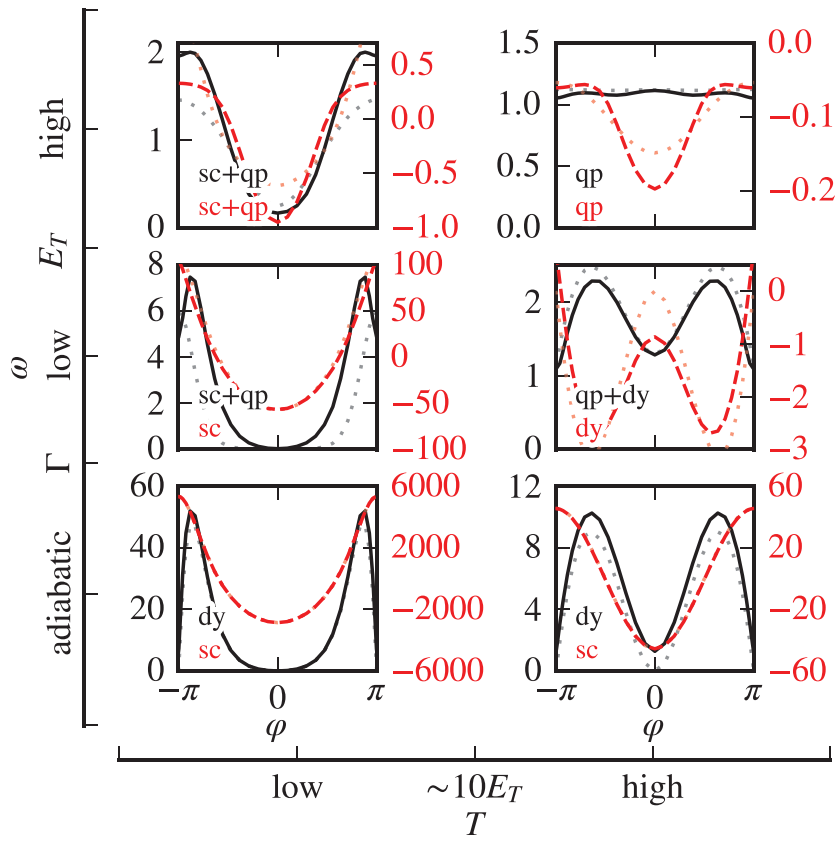

FIG. 1. (Color online) Phase dependence of the admittance $Y$ in units of the normal-state conductance $G_{N}$ (dissipative part, solid; reactive part, dashed), in different regimes of interest. The "low"temperature results have been calculated for $T=E_{T}$; the "high"temperature results, for $T=16 E_{T}$. The adiabatic frequency is $\omega=$ $E_{T} / 200 \ll \Gamma=E_{T} / 20$, the low frequency is $\omega=E_{T} / 4 \gg \Gamma$, and the high frequency is $\omega=10 E_{T}$. Dotted lines show the contribution from dominant parts- $Y_{\mathrm{sc}}$ [Eqs. (1), (10a)]; $Y_{\mathrm{dy}}$ [Eq. (7)]; $Y_{\mathrm{qp}}$ [Eqs. (9), (10c)] -indicated in the lower left corners. The adiabatic-frequency results are obtained from analytical approximations; the others, from a numerical solution of Eqs. (2).

$A(t)=A_{0} \cos (\omega t), A_{0}=-\delta \phi /(2 L)$. Requiring charge neutrality leads to a finite position-dependent scalar potential $U$, but self-consistent numerical solutions to Eqs. (2) indicate that this can be disregarded for $\omega \lesssim 10 E_{T}$.

In the following, our aim is to relate the contributions [Eqs. (4a)-(4c)] to quantities that can be calculated in the absence of the ac drive. ${ }^{9}$ The regimes where the different contributions are relevant depend on the particular values of the phase difference, frequency, and temperature. The main results are summarized in Fig. 1, which shows the phase dependence of the admittance in different regimes of frequencies and temperatures.

\section{A. Low frequency}

For frequencies satisfying $\Gamma \ll \omega \ll E_{g}(\varphi)$, the superconducting correlations follow the time-dependent phase difference, but the electron energy distribution is driven out of equilibrium. Under this constraint, the part of the admittance related to the ac supercurrent [Eq. (4a)] reduces to Eq. (1), i.e., $Y_{\mathrm{sc}} \simeq-2 i \partial_{\varphi} I_{S}(\varphi) / \omega$. Since the supercurrent $I_{S}$ decays exponentially as the temperature increases, this contribution to reactance becomes unimportant at high temperatures $(T \gtrsim$ $\left.10 E_{T}\right)$, unless the frequency is very low.

The second major contribution to reactance comes from a dynamic variation of the population, as given by $Y_{\mathrm{dy}}$. For this, the time-dependent component $\hat{h}_{01} \equiv h_{L}^{\prime}+\hat{\tau}_{3} h_{T}^{\prime}$ of 
the distribution function needs to be solved with a kinetic equation. If we assume again simple time dependence for the superconducting correlations, the first harmonic of the Usadel kinetic equation reads [compare Eqs. (2a)-(2f) and Ref. 6]

$$
\begin{gathered}
D \partial_{x} \cdot\left(\mathcal{D}_{L} \partial_{x} h_{L}^{\prime}-\mathcal{T} \partial_{x} h_{T}^{\prime}+j_{S} h_{T}^{\prime}\right) \\
=\frac{i A_{0}}{2}\left(j_{S}+\partial_{x} \mathcal{T}\right)\left(h_{11}-h_{00}\right)+i(\omega-2 i \Gamma) N h_{L}^{\prime}, \\
D \partial_{x} \cdot\left(\mathcal{D}_{T} \partial_{x} h_{T}^{\prime}+\mathcal{T} \partial_{x} h_{L}^{\prime}+j_{S} h_{L}^{\prime}\right)=i(\omega-2 i \Gamma) N h_{T}^{\prime} .
\end{gathered}
$$

Here $\mathcal{D}_{L / T}$ are the spectral heat/charge diffusion coefficients, $\mathcal{T}$ is an anomalous kinetic coefficient, and $N$ is the local density of states. These quantities are related to the equilibrium retarded Green's function, $\hat{g}^{R}(E)=$ $g(E) \hat{\tau}_{3}+f(E)\left(\hat{\tau}_{x}+i \hat{\tau}_{y}\right) / 2-\tilde{f}(E)\left(\hat{\tau}_{x}-i \hat{\tau}_{y}\right) / 2$, e.g., $j_{S}=$ $\operatorname{Im} j_{E}, j_{E}=\frac{1}{2 i} \operatorname{Tr} \hat{\tau}_{3} \hat{j}_{00}^{R}, N=\operatorname{Re} g$, and $\mathcal{D}_{T}=\frac{1}{2}\left[1+|g|^{2}+\right.$ $\left.|f|^{2} / 2+|\tilde{f}|^{2} / 2\right]$.

At low frequencies, Eqs. (5a) and (5b) should be solved with Andreev reflection boundary conditions amounting to $h_{T}^{\prime}=$ 0 and $\partial_{x} \cdot h_{L}^{\prime} \hat{n}=0$ at the two N/S interfaces. The resulting function $h_{T}^{\prime}$ is in the vector potential gauge finite but small, and as a first approximation we can disregard it. Moreover, gradients of $h_{L}^{\prime}$ are small because of Andreev reflection, and we get a fairly good estimate for the average $h_{L}^{\prime}$ by averaging Eqs. (5a) and (5b) over the normal-metal junction, defining $\langle\cdot\rangle=\int_{0}^{L} d x \cdot / L$. As a result, we get

$$
\hat{h}_{01} \approx\left\langle h_{L}^{\prime}\right\rangle \approx \frac{A_{0}}{2} \frac{j_{S}\left(h_{11}-h_{00}\right)}{(\omega-2 i \Gamma)\langle N\rangle} .
$$

Substituting this into Eq. (4b) and assuming $\omega \lesssim E_{T}$,

$$
\begin{aligned}
Y_{\mathrm{dy}} \equiv & \frac{-i G_{N}}{\omega-2 i \Gamma} \frac{E_{T}}{T} Q(\varphi, T) \\
& \approx \frac{-i G_{N}}{\omega-2 i \Gamma} \int_{-\infty}^{\infty} d E \frac{j_{S}^{2}}{4 T\langle N\rangle \cosh ^{2}[E /(2 T)]} .
\end{aligned}
$$

This is similar to a correction to the dc conductance described in Ref. 10. Its origin can be understood as follows ${ }^{11}$ : the current is carried by a dense spectrum $\left\{\epsilon_{n}(\varphi)\right\}$ of discrete bound states with populations $f_{n}, j(\varphi)=\sum_{n}\left(\partial_{\varphi} \epsilon_{n}\right) f_{n}\left(\epsilon_{n}\right)$. With ac bias one finds $\delta j / \delta V \propto \sum_{n} \partial_{\varphi}^{2} \epsilon_{n} f_{n} / i \omega+\sum_{n}\left(\partial_{\varphi} \epsilon_{n}\right)^{2} \partial_{\epsilon} f_{n} / i \omega$, where the first term is equivalent to $Y_{\mathrm{sc}}$ and the second one to $Y_{\mathrm{dy}}$, as $j_{S} \sim \sum_{n} \delta\left(E-\epsilon_{n}\right) \partial_{\varphi} \epsilon_{n} \sim N \partial_{\varphi} \epsilon$.

The dynamic contribution is purely dissipative and constant for $\omega \ll \Gamma$, contains both reactive and dissipative components for $\omega \approx \Gamma$, and becomes purely reactive and decays for $\omega \gg \Gamma$, as Fig. 1 shows. In general, $\Gamma$ also depends on the phase difference, ${ }^{12}$ so the phase-dependent response at frequencies of the order of the inelastic scattering rate may be quite complicated. On the other hand, Eq. (7) offers a way to probe such phase-dependent scattering rates via an admittance measurement.

The function $Q(\varphi, T)$ is shown in Fig. 2(a) for different temperatures. At $T \gg E_{T}$ the response can be fitted with the function $Q(\varphi) \approx 8.9\left[|\operatorname{saw}(\varphi)|-0.32 \sin ^{2}(\varphi / 2)\right]|\sin (\varphi)|$, where $\operatorname{saw}(\varphi)=(\varphi+\pi \bmod 2 \pi)-\pi$. At low temperatures, $Q(\varphi, T)$ is suppressed for phases at which $T<E_{g}(\varphi)$. Note that $Q$ is positive definite, has almost a double periodicity compared to the kinetic-inductance term, and has a minimum around $\varphi=0$, where the kinetic inductance is at maximum.
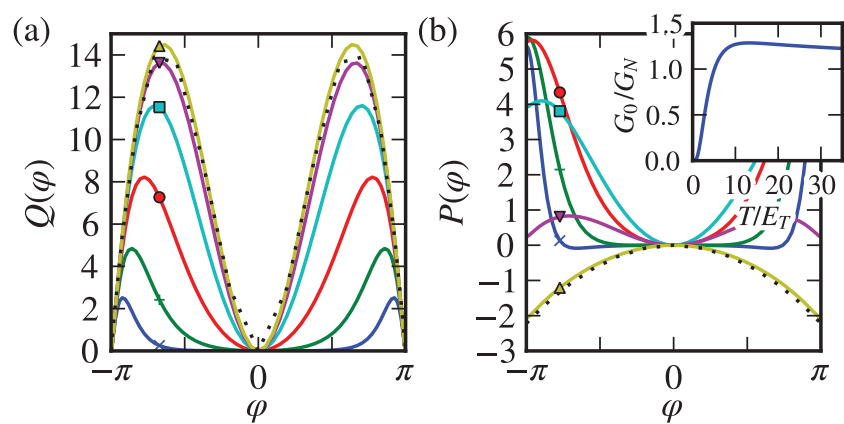

FIG. 2. (Color online) (a) Function $Q(\varphi, T)$ [see Eq. (7)] describing the phase-dependent dynamic contribution to the reactive response. (b) Function $P(\varphi, T)$ [see Eq. (9)] describing the phase dependence of the dissipative part of the admittance at low frequencies. From top to bottom in (a) and marked with symbols in (b): $T / E_{T}=16(\triangle), 8(\nabla), 4(\square), 2(\circ), 1(+), 0.5(\times)$. The dotted lines represent the analytic high-temperature approximations to which $Q$ and $P$ tend for $T \gg E_{T}$. The inset of (b) shows the temperature dependence of $G_{0}(T)$.

The dissipative part of the impedance originates from two additional sources: the quasiparticle part $Y_{\mathrm{qp}}$ and, importantly at low temperatures, a part of the ac supercurrent oscillating in phase with the voltage. The quasiparticle contribution is easiest to derive in a gauge where the vector potential vanishes. Then, $h_{T}^{\prime}$ in Eqs. (5a) and (5b) has the boundary conditions $h_{T}^{\prime}(x= \pm L / 2)= \pm \frac{i \delta \phi}{4}\left(h_{11}-h_{00}\right)$, and solving Eq. (5) while neglecting $h_{L}^{\prime}$ and the right-hand side yields

$$
\begin{gathered}
Y_{\mathrm{qp}}+\operatorname{Re} Y_{\mathrm{sc}} \simeq G_{N} \int_{-\infty}^{\infty} d E \frac{K_{0}(E)}{4 T \cosh ^{2}(E / 2 T)} \\
\equiv G_{0}(T)+\frac{G_{N} E_{T}}{T} P(\varphi, T),
\end{gathered}
$$

where we assumed $\omega \lesssim E_{T}$ and defined $G_{0}(T)$ as the $\varphi=0$ value $[P(0, T)=0]$. The kernel is ${ }^{13} K_{0} \approx\left\langle\mathcal{D}_{T}^{-1}\right\rangle^{-1}-$ $\operatorname{Re} \partial_{\varphi} j_{E} \sim\left\langle N^{2}+\frac{1}{4}\left|f+\tilde{f}^{*}\right|^{2}\right\rangle$. It describes the spectrum of excitations in the junction available for receiving energy: this has a minigap $E_{g}(\varphi)$, so for $\omega, T<E_{g}(\varphi)$, all dissipation vanishes. Note that the appearance of the minigap is related to the presence of the ac supercurrent contribution, $Y_{\mathrm{qp}} \geqslant G_{N}$, and is similar to the usual proximity-enhanced conductance.

The functions $P(\varphi, T)$ and $G_{0}(T)$ are shown in Fig. 2(b). At low temperatures, the temperature and phase dependence shows a clear signature indicating the presence of a minigap in the density of states; note that this also applies to the $Y_{\mathrm{dy}}$ contribution. The dissipation is concentrated at phase differences close to $\pi$, where the minigap is small. In the high-temperature limit, the dissipative term consists of a phase-independent contribution $G_{0}(T)$, and the phase-dependent part has a simple phase and temperature dependence: $-0.23 \operatorname{saw}(\varphi)^{2} G_{N} E_{T} / T$.

\section{B. High frequency}

When the frequency becomes of the order of the Thouless energy $E_{T}$, the above semiadiabatic expressions break down as the spectral quantities become frequency dependent. Starting from Eqs. (4a)-(4c) and following essentially the same steps as above, we can, however, construct approximations valid also 
at higher frequencies. The supercurrent contribution is fairly approximated by

$$
\begin{aligned}
Y_{\mathrm{sc}} \approx & \frac{\sigma_{N} S}{4 \omega} \int_{-\infty}^{\infty} d E\left\{\partial_{\varphi}\left[j_{E}(E)+j_{E}(E+\omega)\right]^{*} h_{00}(E)\right. \\
& \left.-\partial_{\varphi}\left[j_{E}(E)+j_{E}(E+\omega)\right] h_{11}(E)\right\},
\end{aligned}
$$

the dynamic contribution $Y_{\text {dy }}$ by

$$
\begin{aligned}
Y_{\mathrm{dy}} \simeq & \frac{i \sigma_{N} S}{8 \omega(\omega-2 i \Gamma)} \int_{-\infty}^{\infty} d E \frac{\left[j_{E}(E)-j_{E}(E+\omega)^{*}\right]^{2}}{\frac{1}{2}\left\langle g(E)+g(E+\omega)^{*}\right\rangle} \\
& \times\left[h_{11}(E)-h_{00}(E)\right],
\end{aligned}
$$

and the quasiparticle part of the impedance by

$$
\begin{aligned}
& Y_{\mathrm{qp}} \simeq G_{N} \int_{-\infty}^{\infty} d E K(E, \omega) \frac{h_{11}(E)-h_{00}(E)}{2 \omega}, \quad(10 \mathrm{c}) \\
& K(E, \omega)= \frac{1}{2}\left\langle\left[ 1+g(E) g(E+\omega)^{*}\right.\right. \\
&\left.\left.+\frac{1}{2} f(E) f(E+\omega)^{*}+\frac{1}{2} \tilde{f}(E) \tilde{f}(E+\omega)^{*}\right]^{-1}\right\rangle^{-1} .
\end{aligned}
$$

At high temperatures, $Y_{\mathrm{sc}}$ is exponentially suppressed even for high frequencies, similar to the equilibrium supercurrent. The dynamic contribution $Y_{\mathrm{dy}}$, on the other hand, can be neglected for $\omega \gg E_{T}$. Consequently, $Y_{\mathrm{qp}}$ dominates for $\omega, T \gg E_{T}$ (see Fig. 1). At low temperatures, both the supercurrent and quasiparticle contributions are important.

The amplitude of the phase dependence in the admittance is illustrated in Fig. 3, up to high frequencies. As the frequency increases, the proximity-induced phase dependence in the reactive and the dissipative components decays as the admittance approaches the constant normal-state value, $Y(\omega, \varphi) \rightarrow G_{N}$. Similarly, the phase dependence vanishes as the temperature increases. At low frequencies, on the other hand, the reactive component diverges because of the Josephson inductance, and the dissipative component is dominated by $Y_{\mathrm{dy}}$, saturating to $\operatorname{Re} \delta Y \sim G_{N} E_{T} / \Gamma\left(\right.$ can be $\left.\gg G_{N}\right)$ at $\omega=0$.
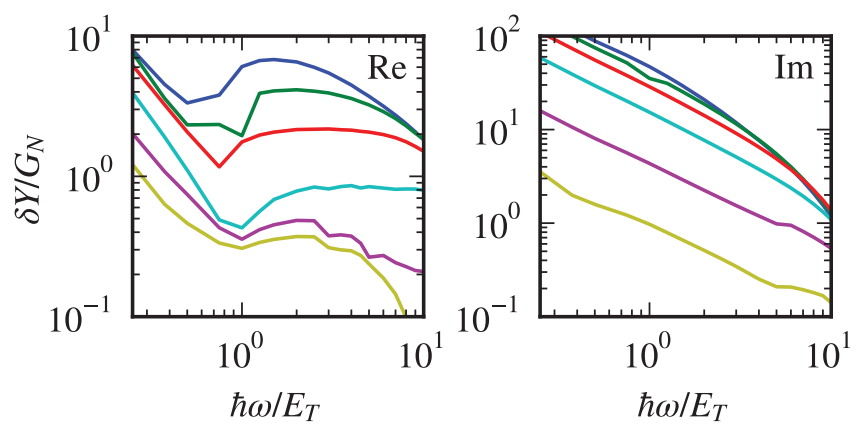

FIG. 3. (Color online) Amplitude $\delta Y=\max _{\varphi}[\operatorname{Re} / \operatorname{Im}] Y(\varphi)-$ $\min _{\varphi}[\operatorname{Re} / \operatorname{Im}] Y(\varphi)$ of the phase dependence in the nonadiabatic dissipative (Re) and reactive ( $\mathrm{Im}$ ) admittance of a long SNS junction, obtained by solving the time-dependent Usadel equations numerically. The lines correspond to temperatures $T / E_{T}=0.5,1,2,4,8,16$ (top to bottom) and were calculated with $\Gamma=0.05 E_{T}$.

\section{DISCUSSION AND CONCLUSIONS}

Above, we computed analytical and numerical results for the ac admittance of SNS junctions. Figures 4 and 1 illustrate that the approximations [Eqs. (10a)-(10d)] reproduce all qualitative features visible in the fully numerical exact solution, and they are quantitatively accurate in a large part of the parameter regime we are interested in. In particular, the deviations between the two approaches are insignificant for frequencies lower than $E_{T}$. At frequencies of the order of $E_{T}$, there are clear quantitative differences in the dissipative component, but the qualitative phase and frequency dependence is the same. This demonstrates that to a fair accuracy the ac admittance can be well described by Eqs. (10a)-(10d) and standard solvers for the equilibrium Usadel equation. In addition, Eq. (10) provides a possibility for making analytical estimates for the admittance contributions.

In a recent experiment probing directly the ac admittance of SNS junctions, ${ }^{11}$ it was found that at frequencies where $\Gamma \ll \omega \ll E_{T}$ (semiadiabatic limit), the reactive response follows closely our prediction consisting of the sum of the Josephson inductance and the dynamic correction [Eq. (7)]. However, both the dissipative contribution and the dependence at high frequencies $\omega \gtrsim E_{T}$ are different: in Ref. 11, the dissipative contribution seems to be directly related to the reactive contribution, and moreover, the amplitude of phase oscillations in susceptibility $\delta \chi=i \omega \delta Y$ decays as frequency increases. The characteristic frequency scale for this was found to be temperature independent and of the order of the Thouless energy. In contrast, in this frequency range our Usadel model predicts $\delta \chi \sim$ const; however, it might be that the simple relaxation time approximation does not include all interaction mechanisms playing a role in the experiment.

Finally, we remark that our work amounts essentially to deriving the parameters for the resistively-shunted-junction model of SNS junctions ${ }^{2}$ : there, the Josephson inductance resulting from the supercurrent term should be modified to include the nonadiabatic correction [Eq. (7)], and the shunt resistor describing the dissipation in the junction should be replaced by the dissipative terms presented in Eqs. (7) and (9).

In conclusion, we described the frequency-dependent admittance of diffusive SNS junctions and showed how the simple adiabatic Josephson inductance picture is modified once the frequency is increased. Besides being of use in studying the dynamics of the system, the detailed frequency dependence can be used to study directly the inelastic scattering rates. Our
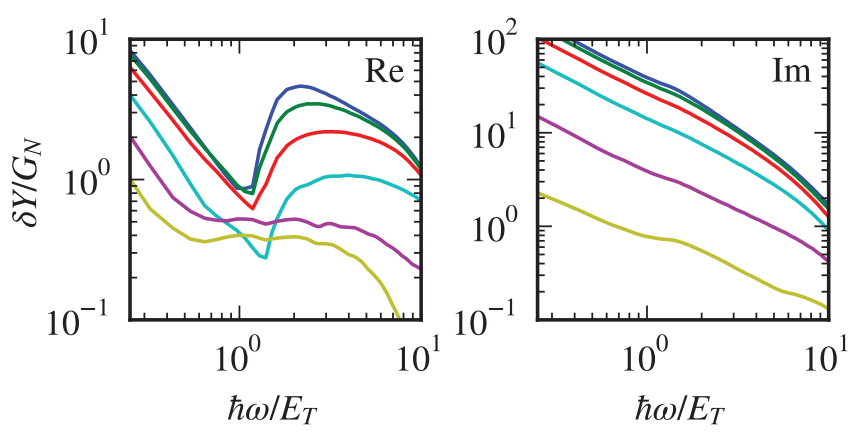

FIG. 4. (Color online) Same as Fig. 3, but results computed from Eqs. (10a)-(10d) rather than numerically. 
results are also relevant for devices utilizing high-frequency properties of SNS junctions, such as those used in metrology and radiation detection.

\section{ACKNOWLEDGMENTS}

We thank H. Bouchiat, S. Gueron, K. Tikhonov, and M. Feigelman for discussions that in part motivated this work, and the Finnish IT Center for Science (CSC), for computer resources. This work was supported by the Academy of Finland, the European Research Council (Grant No. 240362-Heattronics), the Spanish Ministerio de Ciencia e Innovación (Contract No. FIS2008-04209), the EmmyNoether program of the Deutsche Forschungsgemeinschaft, and the Spanish National Research Council (Intramural Project No. 200960I036).
${ }^{1}$ N. Ashcroft and N. Mermin, Solid State Physics (Saunders CollegePhiladelphia, 1976).

${ }^{2} \mathrm{M}$. Tinkham, Introduction to Superconductivity, 2nd ed. (McGrawHill, New York, 1996).

${ }^{3}$ A. A. Golubov, M. Y. Kupriyanov, and E. Il'ichev, Rev. Mod. Phys. 76, 411 (2004).

${ }^{4}$ J. Tucker and M. Feldman, Rev. Mod. Phys. 57, 1055 (1985).

${ }^{5}$ J. M. Warlaumont, J. C. Brown, T. Foxe, and R. A. Buhrman, Phys. Rev. Lett. 43, 169 (1979); M. Fuechsle, J. Bentner, D. A. Ryndyk, M. Reinwald, W. Wegscheider, and C. Strunk, Phys. Rev. Lett. 102, 127001 (2009); L. G. Aslamazov and S. V. Lempitskii, Sov. Phys. JETP 55, 967 (1982).

${ }^{6}$ P. Virtanen, T. T. Heikkilä, F. S. Bergeret, and J. C. Cuevas, Phys. Rev. Lett. 104, 247003 (2010).
${ }^{7}$ K. D. Usadel, Phys. Rev. Lett. 25, 507 (1970); W. Belzig, F. K. Wilhelm, C. Bruder, G. Schön, and A. D. Zaikin, Superlattices Microstruct. 25, 1251 (1999).

${ }^{8}$ J. C. Cuevas, J. Hammer, J. Kopu, J. K. Viljas, and M. Eschrig, Phys. Rev. B 73, 184505 (2006).

${ }^{9}$ These quantities may be calculated for example with the Usadel solver publicly available at [http://ltl.tkk.fi/ theory/usadel1/].

${ }^{10}$ S. V. Lempitskii, Sov. Phys. JETP 58, 624 (1983).

${ }^{11}$ F. Chiodi, M. Ferrier, K. Tikhonov, P. Virtanen, T. Heikkilä, M. Feigelman, S. Guéron, and H. Bouchiat, (2010), e-print arXiv:1005.0406.

${ }^{12}$ T. T. Heikkilä and F. Giazotto, Phys. Rev. B 79, 094514 (2009).

${ }^{13}$ The latter approximation is the one used in Ref. 6 and captures the minigap correctly, whereas the former gives a better approximation to the integral but is inaccurate at energies $E \lesssim E_{g}(\varphi)$. 